\title{
Plasma irisin and its associations with oxidative stress in athletes suffering from overtraining syndrome
}

\author{
R. JORO ${ }^{1}$, A. KORKMAZ ${ }^{1}$, T.A. LAKKA ${ }^{1,2,3}$, A.L.T. UUSITALO ${ }^{\dagger 4,5}$ and \\ M. ATALAY ${ }^{\dagger 1 *}$ (1)
}

${ }^{1}$ Institute of Biomedicine, School of Medicine, University of Eastern Finland, Kuopio Campus, Finland

${ }^{2}$ Department of Clinical Physiology and Nuclear Medicine, Kuopio University Hospital, Kuopio, Finland

${ }^{3}$ Foundation for Research in Health Exercise and Nutrition, Kuopio Research Institute of Exercise Medicine, Kuopio, Finland

${ }^{4}$ Department of Sports and Exercise Medicine, Clinicum, University of Helsinki, Helsinki, Finland

${ }^{5}$ Clinic for Sports and Exercise Medicine, Foundation for Sports and Exercise Medicine, Helsinki, Finland

Received: February 13, 2020 • Accepted: June 03, 2020

Published online: December 31, 2020

(c) 2020 Akadémiai Kiadó, Budapest

\begin{abstract}
Irisin is a novel exercise-induced myokine that may be involved in regulating energy metabolism. We determined whether overtraining syndrome (OTS) and its biochemical markers are associated with plasma irisin levels in athletes. Seven severely overtrained athletes (OA) and 10 healthy control athletes (CA) were recruited and examined at the time of diagnosis (baseline) and after 6- and 12-months follow-up. Training volume and intensity were initially restricted but progressively increased in OA as OTS symptoms alleviated; CA continued their normal training routine. A maximal cycle ergometer test was performed with irisin analyzed before and after the test. Before the exercise test, irisin levels tended to be lower in OA than in CA at baseline $(154.5 \pm 28.5$ vs. $171.7 \pm 58.7 \mathrm{ng} / \mathrm{mL})$. In both groups, at rest irisin levels changed only marginally during follow-up and were not affected by maximal exercise, nor were they associated with physical performance or body fat percentage. Irisin concentration at rest correlated positively with an oxidative stress marker, malondialdehyde (MDA) and negatively with an antioxidant protection marker, oxygen radical absorbance capacity (ORAC) in response to the exercise test in OA at baseline. Our findings
\end{abstract}

\footnotetext{
*Corresponding author. E-mail: mustafa.atalay@uef.fi

${ }^{\dagger}$ These two authors contributed equally to this work.
} 
help to clarify the possible contribution of irisin and its association with oxidative stress in the pathophysiology of OTS.

\section{KEYWORDS}

overtraining, recovery, exercise, myokine

\section{INTRODUCTION}

Overtraining syndrome (OTS) in athletes is a chronic physical stress condition that remains a diagnostic challenge due to the lack of reliable parameters for the evaluation of OTS or for monitoring recovery from OTS [1]. Although multiple mechanisms have been proposed, the pathophysiology of OTS and the associated long-term performance impairment is not entirely clear [2-6]. Putative pathophysiological mechanisms include impairments in skeletal muscle carbohydrate and lipid metabolism [4], increased oxidative stress [6, 7], muscle microtraumainduced chronic inflammation [5], and inappropriate production and activities of cytokines such as interleukin-6 (IL-6) [8], as well as hormonal alterations [9-12]. Notably, the interaction between different mechanisms related to the pathogenesis of OTS have not been elucidated.

Some novel clinical and biochemical markers of OTS have recently been explored, and new pathophysiological mechanisms have been proposed, although they need further confirmation. OTS is inherently severe and therefore, the main difference between OTS and overreaching states is a lack of recovery after a period of resting, and the duration and severity of the symptoms. While OTS has been postulated to be related to strenuous resistance training [13], in a latest series of reports by Cadegiani and Kater [10,11,14] OTS was postulated to be induced by energy imbalance, independent of excessive training. A major outcome of these studies was that OTS could result in "paradoxical deconditioning" [11]. Notably, OTS could be indicated by lower salivary cortisol levels 30 minutes after waking up, which can be correlated negatively with fatigue $[9,10,14]$. However, these studies also had certain limitations: control subjects had a broad age range, and the analyzed parameters were not adjusted for the age difference in the statistical analyses. Furthermore, in several of the correlation analyses a very heterogeneous subject group was utilized, consisting of both overtrained athletes and healthy control persons [10]. Nonetheless, a recent systematic review of 22 studies on OTS revealed that no diagnostic evidence other than unexplained decrement in physical performance was verified as a reliable marker of OTS, particularly in resistance training [13].

Irisin is a novel myokine and adipokine that has been found to increase adipocyte browning in subcutaneous fat, as well as promoting thermogenesis regulated by uncoupling protein 1 (UCP1) and resting energy expenditure in rodents $[15,16]$. Irisin has also been reported to be released from contracting skeletal muscle during acute exercise in a manner that is dependent on both the intensity and duration of the exercise [17-21]. Whereas acute exercise has been shown to increase plasma irisin levels in some studies [20,21], no such effect has been evident in others $[22,23]$. Although analytical studies have confirmed the existence of irisin in humans, as well as its presence in circulation and its ability to be regulated by exercise [20, 24], the acute and chronic effects of exercise-related irisin expression on skeletal muscle metabolism are not known 
in detail, as has been reviewed by Fatouros [25]. Huh et al. [26] demonstrated that irisin directly stimulated MAPK (mitogen-activated protein kinase) in human myotubes in vitro. As the MAPK pathway has a key role in energy substrate regulation in skeletal muscle during exercise, irisin may exert direct effects on skeletal muscle metabolism. A meta-analysis [27] demonstrated that chronic exercise training tended to lower plasma irisin levels, which may be the result of a reduction in the body fat content [28]. In addition, a recent study among young amenorrheic athletes suggested that low plasma irisin levels reflected a state of chronic energy deficiency [29]. Therefore, we postulated that during OTS, a dysregulation of energy metabolism would also affect irisin levels, which could be utilized as a marker in OTS diagnosis.

We earlier reported using the same experimental setting that oxidative stress may exert an impact on the pathophysiology of OTS [6] and, furthermore, that a pro-inflammatory cytokine response to acute exercise may represent a chronic maladaptation state in overtrained athletes [3]. Notably, impairments in antioxidant defense in OTS may reflect an overall regulatory decompensation status of the organism [7]. Although the role of irisin in redox regulation and in the control of oxidative stress and inflammation have recently been explored in different animal models [30-32], data from exercise models, especially those mimicking OTS are still lacking.

As far as we are aware, the associations between severe OTS or other chronic physical stress conditions and irisin have not been investigated. We therefore compared plasma irisin levels before and after a maximal exercise test in athletes suffering from OTS as well as their peers without OTS. Furthermore, inflammation and oxidative stress, which are controlled by irisin, are postulated to be predisposing factors for OTS. Therefore, in order to evaluate the link between irisin and oxidative stress/inflammation and with an effort to obtain more evidence on the pathogenesis of OTS, we analyzed the association of irisin with oxidative stress, antioxidant protection markers and inflammatory cytokines. We also studied how recovery from OTS would affect plasma irisin levels during 12 months of follow-up in an effort to evaluate whether irisin would be a useful marker for monitoring recovery from exercise as well as assisting in the early diagnosis of OTS in elite athletes.

\section{MATERIALS AND METHODS}

\section{Participants}

Seven over-trained athletes (OA, two females and five males) and 10 healthy control athletes (CA, five females and five males) were investigated in this study. The athletes participated in various endurance sports and were recruited when they responded to a nationwide advertisement. All athletes were competing at the Finnish national level and reported no history of smoking. The criteria for OTS diagnosis were: (1) An unexplained decrement in physical performance and fatigue despite a recovery period of at least three weeks; (2) progressively increased training volume and intensity for up to six months prior to the onset of OTS symptoms; (3) the continuation of training without a sufficient recovery time; and (4) no reports of chronic or recent other illnesses explaining the symptoms, i.e., the possible illnesses and diseases were carefully medically ruled out in University Hospital $[6,33,34]$. The criteria were confirmed by interviewing the athletes and their coaches along with an examination of training logbooks. An experienced physician performed extensive clinical examinations with specific laboratory tests intended to clarify the background of the symptoms of every athlete. 
The overtraining state of the present subjects was also related to higher perceived stress and disturbed cardiac autonomic modulation detected as attenuated cardiac parasympathetic modulation after awakening [33], during active orthostatic test (standing up) and in response to cognitive task (Stroop Color Word Test) and to relaxation after Stroop tasks [35]. Autonomic dysfunction in the overtrained athletes participating in the present study was further demonstrated by a stronger relationship between RRi and vagally mediated RRi variability during $24-\mathrm{h}$ ECG recording compared to control athletes [36]. This relationship was normalized after 6 months of recovery from OTS [36]. Notably, severely overtrained athletes displayed a lower cognitive performance compared to the controls. Moreover, the same OT athletes participating in our study were reported to be moderately depressed according to their scores in both the standardized Hamilton and the Montgomery-Åsberg Depression Rating Scales, although their brain serotonin uptake levels did not display any changes compared to the control athletes [34]. Furthermore, at the time of diagnosis, at rest the overtrained athletes had higher levels of plasma protein carbonyls, a biomarker of oxidative stress, than the controls [6].

The female athletes had regular menses except for one CA female who had athletes' amenorrhea symptoms. One OA female had e-pills, two of five CAs had e-pills and the CA female with amenorrhea had progesterone pills. One male athlete in OA had sleeping pills and antidepressant from 6 to 12 months of recovery from OTS.

The participants were informed of the study protocol that had been approved by the Research Ethics Committee of the Hospital District of Northern Savo, and they all provided a written consent to participate in the study.

\section{Follow-up}

During the 12 months of follow-up, the OA took part in supervised low-to-moderate intensity exercise training to ensure a full recovery and thereafter an increase in physical performance. CA continued their normal training routine. All athletes were examined at the time of diagnosis (baseline) and after 6 and 12 months of follow-up. The OA were also examined after one month of follow-up, but only the basic characteristics of the participants at this time point are shown, and the one-month plasma measurements were not included in the statistical analyses. At each study visit, OA were examined to determine whether they still displayed signs of OTS $[6,33,34]$. The athletes were examined $2 \mathrm{~h}$ after a light breakfast, and were instructed to abstain from strenuous physical activity on the day before the examinations and not to drink coffee, tea, chocolate, or cola drinks on the examination morning or the previous evening. The athletes were also instructed not to drink alcohol $48 \mathrm{~h}$ before the examinations.

\section{Experimental testing}

Cardiorespiratory fitness was assessed by a maximal exercise test supervised by a physician and performed to volitional exhaustion using the Ergoline Ergo-metrics 900 bicycle ergometer (Ergoline, Bitz, Germany). To define peak oxygen consumption $\left(\mathrm{VO}_{2}\right.$ peak $)$ due to the graded type of exercise test conducted on the bicycle ergometer, pulmonary gas exchange values were measured by the breath-by-breath method using the SensorMedix $V_{\max } 29$ analyzer (CareFusion, San Diego, California, USA). The analyzer was calibrated before each test and was verified immediately after every test. Heart rate was recorded using the Marquette Hellige electrocardiography (ECG) recorder (GE Medical Systems, Freiburg, Germany), and the rating 
of perceived exertion was evaluated with the Borg scale. Standard physiological criteria were used to determine maximal effort (American College of Sports Medicine, 2001). The clinical examination performed by a physician also excluded the possibility that cardiac, lung and muscle pathologies lay behind the underperformance. Body fat percentage and lean body mass were measured by bioelectrical impedance analysis using the InBody 3.0 body composition analyzer (Biospace, Seoul, South Korea).

\section{Biochemical Indices}

Blood samples taken at rest and immediately after exercise were collected from an antecubital vein into lithium-heparin tubes and centrifuged immediately after collection at $1,200 \mathrm{~g}$ and $4{ }^{\circ} \mathrm{C}$ for 15 min to separate the plasma. Plasma samples were stored in multiple portions at $-80{ }^{\circ} \mathrm{C}$ until analysis. Samples were thawed only once for the measurement of oxidative stress and antioxidant markers [6]. Plasma irisin levels were measured using ELISA kits (Phoenix Pharmaceuticals Inc, Belmont, CA, USA) according to the manufacturer's protocol. According to our analyses, the intra-assay variation was $3.90 \%$ and the inter-assay variation was $7.57 \%$. Because of the sigmoidal character of the concentration changes in response to different standard concentrations and different sample dilutions, special caution was taken to determine optimal sample dilution in order for the absorbance values to be in the region of maximal linearity. In our measurements we achieved readings that corresponded to the linear part of the standard curve. IL-6, TNF- $\alpha$, IL-10, IL-1 $\beta$, leptin, IGF-1, oxygen radical absorbance capacity (ORAC), total malondialdehyde (MDA) and nitrotyrosine levels were measured as previously described $[15,37]$.

\section{Statistical analysis}

The statistical analyses were performed using the IBM SPSS Statistics software, Version 19.0 (IBM Corporation, Armonk, New York, USA). The normality of the residuals was inspected visually from histograms. Homogeneity of variance was inspected by plotting residuals against predicted values. Non-parametric tests were used for analyzing the data with non-normal distribution. Pearson's coefficients for correlation were calculated for normally distributed variables. The groups were compared with the linear mixed-effects models using group, time, exercise, and sex as fixed factors. Body fat percentage was also included in the model. Association of irisin levels with IL-6, TNF- $\alpha$, IL-10, IL- $1 \beta$, leptin, IGF-1, MDA, ORAC and nitrotyrosine was assessed by Pearson's correlation test for normally distributed variables and by Spearman's correlation test for skewed variables. The results are presented as mean \pm standard deviation (SD). Differences with $P$-values $<0.05$ were considered statistically significant.

Statistical power analysis was performed by GLIMMPSE software [38]. Varying results from the previous heterogenic study designs complicate the power analysis. If we were to detect similar (approximately 1.2-fold) post-exercise increases in irisin concentration as observed in some previous studies involving trained $[21,26]$ and untrained individuals [18], then we would achieve power $>80 \%$ (alpha $=0.05$ ). In order to achieve power $>80 \%$ in detecting betweengroup differences of the same magnitude, a total $N$ of approximately 20-30 would have been needed depending on the expected variability. In fact, our observed power was a reasonable, although modest, $60 \%$. 


\section{RESULTS}

\section{Basic participant characteristics}

The basic characteristics of the participants are presented in Table 1. Fat percentage was higher in OA regardless of the gender as we have previously reported [3]. During one month of follow-up, $\mathrm{VO}_{2 \text { peak }}$ decreased in OA but not in CA (Table 1). Only three OA were able to exercise according to the programmed training schedule. The other OA performed light physical activity 2-4 times per week to encourage their recovery. $\mathrm{VO}_{2 \text { peak }}$ increased in all OA except one athlete during the follow-up, but the main effect of time remained statistically non-significant in both groups and there were no differences between groups (Table 1). OTS symptoms became exacerbated in one male OA. In another male $\mathrm{OA}, \mathrm{VO}_{2 \text { peak }}$ increased but his sport-specific symptoms did not resolve during the follow-up. Changes in heart rate were statistically non-significant in both groups (Table 1). Three OA dropped out before the last measurement, with the reason being that they were experiencing a worsening of their OTS symptoms.

\section{Plasma irisin levels}

Initial plasma irisin levels were slightly lower in OA when compared to CA at rest $(154.5 \pm 28.5$ vs. $171.7 \pm 58.7 \mathrm{ng} / \mathrm{mL}, P>0.050)$. Irisin concentrations at rest did not change during the follow-up in either group (Fig. 1). The increase in irisin in response to acute exercise was higher in OA than in CA at the 6 months' follow-up $(19.0 \pm 69.3$ vs. $8.1 \pm 46.4 \mathrm{ng} / \mathrm{mL}, P>0.050)$. There was no statistically significant effect of time on the irisin level during the follow-up or in the irisin response to acute exercise in CA or OA. Neither irisin levels at rest nor the irisin response to exercise was statistically significantly correlated with OTS, recovery from OTS, or any change in aerobic performance. Fat percentage was not associated with either irisin levels at rest or in the irisin responses to acute exercise. Interestingly, lean body mass correlated negatively with the irisin concentration in $\mathrm{OA}(r=-0.749 ; P<0.001)$ whereas no correlation was detected in CA.

Table 1. Basic characteristics (mean \pm standard deviation) of overtrained athletes (OAs) and control athletes (CAs) at the time of diagnosis (0 month) and during 12-month follow-up

\begin{tabular}{lcccccc}
\hline Group & $\begin{array}{c}\text { Time } \\
(\text { month })\end{array}$ & $\begin{array}{c}\text { Height } \\
(\mathrm{cm})\end{array}$ & $\begin{array}{c}\text { Body } \\
\text { weight }(\mathrm{kg})\end{array}$ & $\begin{array}{c}\text { Body } \\
\text { fat\% }(\%)\end{array}$ & $\begin{array}{c}\mathrm{VO}_{2 \text { peak }} \\
(\mathrm{l} / \mathrm{min})\end{array}$ & $\begin{array}{c}\text { Maximal heart } \\
\text { rate }(1 / \mathrm{min})\end{array}$ \\
\hline CA & & $171.1 \pm 6.7$ & $64.9 \pm 10.3$ & $15.6 \pm 7.2$ & $3.8 \pm 0.9$ & $192 \pm 6$ \\
& 6 & & $64.4 \pm 6.1$ & $16.9 \pm 8.6$ & $4.0 \pm 0.8$ & $192 \pm 8$ \\
OA & 12 & & $64.5 \pm 6.1$ & $15.6 \pm 7.9$ & $3.9 \pm 0.8$ & $190 \pm 4$ \\
& 0 & $176.7 \pm 9.0$ & $71.6 \pm 10.3$ & $17.2 \pm 7.9$ & $3.8 \pm 0.9$ & $190 \pm 10$ \\
& 1 & & - & - & $3.6 \pm 0.9^{*}$ & $179.7 \pm 13$ \\
& 6 & & $74.8 \pm 7.5$ & $15.9 \pm 4.2$ & $4.2 \pm 1.0$ & $183 \pm 14$ \\
& 0 & & $75.0 \pm 5.3$ & $19.6 \pm 13.0$ & $4.2 \pm 1.0$ & $183 \pm 12$ \\
\hline
\end{tabular}

Values are means $\pm \mathrm{SD}$. ${ }^{*}$ Difference in $\mathrm{VO}_{2 \text { peak }}$ during the first month compared to the time of diagnosis in OA (Wilcoxon signed-rank test ${ }^{*} P=0.028$ ). 


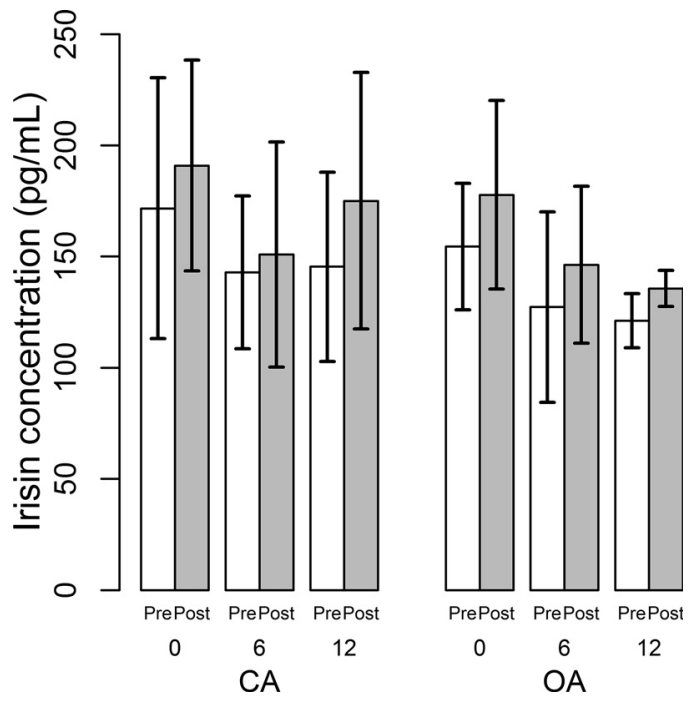

Fig. 1. Plasma irisin levels (mean \pm standard deviation) before (PRE) and after (POST) exercise test at baseline and during the follow-up in control (CA) and overtrained athletes (OA)

\section{Association of irisin with oxidative stress, antioxidant markers, and inflammatory cytokines}

At baseline we observed a statistically significant positive correlation between the irisin level at rest and the change in an oxidative stress marker, MDA, in the athletes' response to the maximal exercise test in OA but not in CA (Fig. 2a). In addition, a strong negative correlation of irisin at

$2 a$

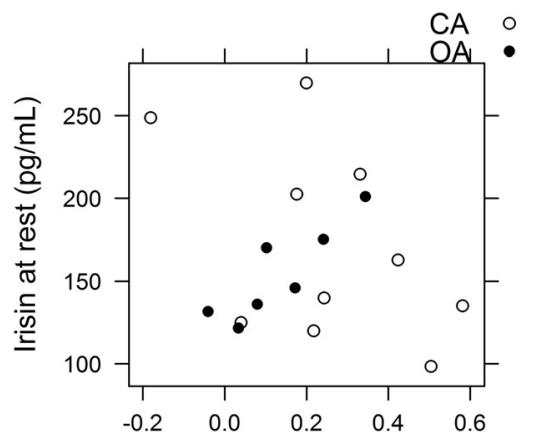

MDA exercise response $(\mathrm{nmol} / \mathrm{mg})$ 2b

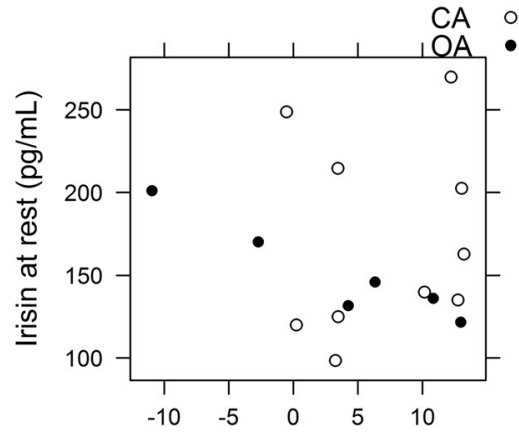

ORAC exercise response $(\mathrm{nmol} / \mathrm{mg})$

Fig. 2. (a) and (b) Plasma irisin levels at baseline in control and overtrained athletes (OA) before exercise correlated positively with the change in malondialdehyde (MDA) concentration after exercise (PRE-POST) $(r=0.878, P=0.010)$ and negatively with the change in oxygen radical absorbance capacity (ORAC)

$$
(r=-0.955, P=0.003)
$$


rest with the change in an antioxidant protection marker, ORAC, during the maximal exercise test was also detected in OA but not in CA (Fig. 2b). Furthermore, at the time of the diagnosis, the plasma level of irisin at rest correlated positively with the TNF- $\alpha$ response to the maximal exercise test in OA $(r=0.793, P=0.033)$. When considering all subjects in the analysis at the 6 months' follow-up, the irisin level at rest correlated positively with the amount of nitrotyrosine both at rest $(r=0.655, P=0.006)$ and after maximal exercise test $(r=0.668, P=0.005)$.

\section{DISCUSSION}

The main finding of this study was that OTS did not correlate with plasma irisin levels among athletes. In addition, decreased training volume and intensity did not influence the plasma concentration of irisin at rest or the irisin response to a single bout of exercise in athletes suffering from OTS, although there was a tendency towards lower irisin levels at the time of diagnosis and progressively decreasing irisin levels in response to acute exercise in OA during the follow-up. On the other hand, at baseline, irisin concentrations at rest correlated positively with changes in oxidative stress and negatively with changes in antioxidant capacity during acute exercise. This is the first study to demonstrate the association of irisin, which is a novel myokine, with acute exercise-induced alterations in oxidative stress and antioxidant protection in humans.

As far as we are aware, plasma irisin levels have not been examined in overload training or in other chronic physical stress conditions. Thus, little is known about the effects of chronic overload training, OTS and impaired recovery from OTS on plasma irisin levels. Exercise intervention studies showed little effect on irisin [20, 22, 23, 39]. However, changes in body composition and nutritional status in response to exercise interventions may affect plasma irisin levels. In fact, whole body energy status and body muscle mass may be more important irisin regulators than exercise per se [39-41], although conflicting information also exists [42, 43]. As energy insufficiency may be one factor in the multi-factorial pathogenesis of OTS, this may hint that irisin, much like leptin, may be a potential marker for the development of chronic performance impairment. In situations where there is a lack of energy intake, plasma irisin levels may decrease and thus reflect the need to conserve energy reserves [29]. On the other hand, the weight loss in response to exercise training may also lead to increased plasma irisin levels. In the present study, we demonstrated a negative correlation of irisin with lean body mass in OA but not in CA, whereas fat percentage was not associated with either irisin levels at rest or irisin responses to acute exercise. Therefore, we cannot conclude that irisin levels are affected directly by a relative increase in muscle mass [37]. Anastakilis et al. [17] detected that lean body mass was positively correlated with irisin in both males and females and that irisin levels were higher in females as compared to males. Despite a similar trend, we did not observe statistically significant differences as males were compared to females. Most notably, in the present study overtrained athletes presented higher body fat percentage than control athletes, and recently Cadegiani and Kater have found similar results in overtrained male athletes [9]. Nevertheless, in our study for the comparison of plasma irisin levels in both groups, linear mixed-effects models were used, where sex and body fat percentage were set as covariates to normalize the effects of these variables on irisin levels. There is a need for further studies to reveal how energy metabolism and its markers are associated with overtraining. 
To date, the acute effects of irisin have not been extensively examined. Irisin may act as a MAPK-activator [26], thus stimulating the same metabolic pathways as IL-6 [44]. The irisin response to acute exercise and exercise training may be attributed to energy depletion and the ability of an individual to restore his/her muscle ATP content. While serum irisin levels can be induced by low ATP levels, the levels return to normal in response to the restoration of muscle ATP content [40]. The current findings are inconsistent, and the relevance of irisin's effect on skeletal muscle metabolism in acute exercise needs further clarification. It seems, though, that short-term intensive acute exercise is capable of elevating serum irisin concentrations [20, 21 , 45]. Since we observed only marginal changes in irisin during acute exercise in both groups and slightly higher plasma irisin levels after exercise in OA than in CA, one may speculate whether a short-term progressive cycle ergometer test is sufficient to elevate plasma irisin levels in athletes, who are fit and well trained. This proposal is supported by Huh et al. [4] who demonstrated that serum irisin levels were increased in untrained but did not change in trained individuals in response to an acute sprint exercise.

One reason for the discrepancy in the literature between the studies on the role of exercise in circulating irisin levels and the physiological effects of irisin in humans may be methodological, i.e., what are the significance of irisin in the systemic circulation and the nature of its detection by immunoassays [20]. To resolve this latter question, an analytical study demonstrated the validity of the immunoassay kit, in which the antibody used showed $100 \%$ cross-reactivity with a soluble irisin precursor FNDC5 (fibronectin type III domain-containing protein 5) [20]. Moreover, the architecture of human irisin was identified and plasma irisin levels were precisely quantified in a previous study that utilized targeted mass spectrometry with control peptides that were enriched with stable isotopes [24].

The diagnosis of OTS is challenging because of the limited availability of biomarkers for this syndrome and the difficulty in their interpretation. In this study, we considered the need of elucidation of different mechanisms related to the pathogenesis of OTS and compared the results with those of our previous publication on OTS in the same subjects where we detected a pro-inflammatory cytokine response to acute exercise in OA as evidenced by an increase in the pro-inflammatory IL- $\beta$ concentration only in OA, whereas the levels of the anti-inflammatory IL-10 were greater in CA [3]. A growing number of studies investigating the association between irisin and inflammation have demonstrated an anti-inflammatory role of irisin in several pathological conditions (for a review see Askari et al. [46]). Here, we showed that at the baseline, irisin at rest correlated with the TNF- $\alpha$ response to a maximal exercise test in $\mathrm{OA}$, suggesting that irisin reacts to unbalanced cytokine metabolism, which might be evident in OTS.

Moreover, we have earlier reported that resting oxidative stress was increased during OTS as demonstrated by higher plasma levels of protein carbonyls in OTS than in controls and by antioxidant protection not reacting to acute exercise-induced stress [6]. Moreover, acute exercise increased total antioxidant capacity as measured by ORAC in controls but not in OA at baseline and six months' follow-up [6]. Margonis et al. [7] found that a 12-week resistance-training period caused a performance decline, interpreted as overtraining, and a marked response in the oxidative stress biomarkers which, in some cases, was proportional to the training load. Impaired antioxidant protection in OTS may be a result of a stage of overall regulatory decompensation in an athlete suffering from OTS. In the present study, we found that plasma irisin levels at rest correlated positively with changes in lipid peroxidation and negatively with 
changes in ORAC in the response to a single bout of exercise at the time of the diagnosis of OTS. Furthermore, after six months of follow-up plasma irisin levels at rest correlated positively with plasma nitrotyrosine concentrations at rest and after acute exercise when including all the subjects in the analysis. The association of irisin with oxidative stress remained unclear for a long time until the recent mechanistic studies on the multifaceted role of irisin in the regulation of redox homeostasis. Irisin is a peroxisome proliferator-activated receptor gamma coactivator 1-alpha- (PGC1 $\alpha$ )-dependent myokine, and PGC- $1 \alpha$ contributes to the control of mitochondrial biogenesis and the regulation of mitochondrial antioxidant enzymes superoxide dismutase and glutathione peroxidase [30]. Recent studies have revealed that the antioxidant role of irisin is mediated mainly through the nuclear translocationmediated nuclear factor (erythroid-derived 2)-like 2 (Nrf2) protein pathways [3, 24]. Nrf2 is generally considered as an essential transcription factor in the protection against oxidative stress by enhancing the expression of various antioxidant genes [31, 32]. Furthermore, independent of the Nrf2 pathway, FNDC5 overexpression or irisin supplementation induced the expression of target proteins of $\mathrm{Nrf} 2$, including heme-oxygenase-1 (HO-1), and consequently it attenuated NADPH oxidase activity, a major source of reactive oxygen species (ROSs) [32]. These results collectively provide evidence for the role of irisin in the modulation of oxidative stress, suggesting that it may be responsive to increased oxidative stress and lower antioxidant capacity.

In addition to the inflammatory and oxidative stress markers, several hormones have been utilized to reveal the pathogenesis and diagnosis of OTS, including testosterone. A number of recent studies point out a tight association between irisin and testosterone, although some controversy exists. A previous study reported that circulating irisin was inversely associated with serum testosterone levels in patients with metabolic syndrome, particularly in the subjects with hypogonadism [47]. In contrast to these results, the same group recently demonstrated a positive association of circulating irisin with serum testosterone, where a testosterone replacement therapy considerably increased serum irisin levels [48]. Likewise, chronic exposure to irisin resulted in increased levels of testosterone levels in male rats [49]. Since we have a group of OTS and control athletes including members of both sexes, and we did not measure testosterone levels in the present study, the relationship between irisin and testosterone remains out of the scope of this report.

Our study provides novel insights into the physiological role of irisin in athletes. In addition, we had the opportunity to evaluate how irisin might associate with OTS. There are a few important restrictions to take into account when interpreting our results. Our study population is small due to the strict diagnostic criteria of OTS, the rarity of true OTS and difficulties in the recruitment of participants. Therefore, the statistical power was limited and some of the differences between the study groups remained statistically non-significant despite the presence of trends in the results. As all of the OA had individual recovery speeds and training programs, it is difficult to evaluate whether or not it was the increasing training volume and intensity after recovery that had affected plasma irisin levels. Furthermore, a more strenuous exercise test might have been needed to evoke a statistically significant irisin response to acute exercise. Importantly, our study is applicable only to the athletes participating in high volume endurance training. Moreover, lack of precise data on diet, energy intake, and energy expenditure of the participants restricts interpretation of the results. 


\section{CONCLUSIONS}

OTS or the recovery from this syndrome among athletes did not correlate either with plasma irisin levels or its response to a single bout of exercise despite a tendency towards lower irisin levels at the time of diagnosis and during the follow-up in OA. Furthermore, changes in the plasma irisin concentration were not associated with the modifications of normal training routine or altered aerobic performance. Nevertheless, our results on the association of plasma irisin levels at rest with increased oxidative stress and the antioxidant capacity in response to acute exercise provide novel evidence on the regulatory role of irisin in the antioxidant defense against exercise-induced oxidative stress, one of the putative mechanisms of OTS pathogenesis. Further research is warranted to clarify the role of irisin in the adaptation to intensive exercise training and OTS.

Conflict of interest: The authors have no conflicts of interest.

\section{ACKNOWLEDGMENTS}

This work was financially supported by grants from the Ministry of Education and Culture of Finland, the University of Eastern Finland, the Finnish Foundation for Research in Sports, and COST Action CA16112. The authors would like to thank Prof. Keith DeRuisseau for assisting with the English language editing of the manuscript and Ms. Taina Vihavainen for the technical assistance.

\section{REFERENCES}

1. Meeusen R, Duclos M, Foster C, Fry A, Gleeson M, Nieman D, et al. Prevention, diagnosis, and treatment of the overtraining syndrome: joint consensus statement of the European College of Sport Science and the American College of Sports Medicine. Med Sci Sports Exerc 2013; 451: 186-205.

2. Fry RW, Morton AR, Keast D. Overtraining in athletes. An update. Sports Med 1991; 121: 32-65.

3. Joro R, Uusitalo A, DeRuisseau KC, Atalay M. Changes in cytokines, leptin, and IGF-1 levels in overtrained athletes during a prolonged recovery phase: a case-control study. J Sports Sci 2017; 3523: 2342-9.

4. Petibois C, Cazorla G, Poortmans JR, Deleris G. Biochemical aspects of overtraining in endurance sports : the metabolism alteration process syndrome. Sports Med 2003; 332: 83-94.

5. Smith LL. Cytokine hypothesis of overtraining: a physiological adaptation to excessive stress? Med Sci Sports Exerc 2000; 322: 317-31.

6. Tanskanen M, Atalay M, Uusitalo A. Altered oxidative stress in overtrained athletes. J Sports Sci 2010; 283: 309-17.

7. Margonis K, Fatouros IG, Jamurtas AZ, Nikolaidis MG, Douroudos I, Chatzinikolaou A, et al. Oxidative stress biomarkers responses to physical overtraining: implications for diagnosis. Free Radic Biol Med 2007; 436: 901-10.

8. Robson-Ansley PJ, Blannin A, Gleeson M. Elevated plasma interleukin-6 levels in trained male triathletes following an acute period of intense interval training. Eur J Appl Physiol 2007; 994: 353-60. 
9. Cadegiani FA, Kater CE. Body composition, metabolism, sleep, psychological and eating patterns of overtraining syndrome: results of the EROS study (EROS-PROFILE). J Sports Sci 2018; 3616: 1902-10.

10. Cadegiani FA, Kater CE. Novel causes and consequences of overtraining syndrome: the EROS-DISRUPTORS study. BMC Sports Sci Med Rehabil 2019; 1121x: eCollection 2019.

11. Cadegiani FA, Kater CE. Novel insights of overtraining syndrome discovered from the EROS study. BMJ Open Sport Exerc Med 2019; 51: e000542,000542. eCollection 2019.

12. Hackney AC. Hypogonadism in exercising males: dysfunction or adaptive-regulatory adjustment? Front Endocrinol (Lausanne) 2020; 1111.

13. Grandou C, Wallace L, Impellizzeri FM, Allen NG, Coutts AJ. Overtraining in resistance exercise: an exploratory systematic review and methodological appraisal of the literature. Sports Med 2020; 504: 815-28.

14. Cadegiani FA, Kater CE. Inter-correlations among clinical, metabolic, and biochemical parameters and their predictive value in healthy and overtrained male athletes: the EROS-CORRELATIONS study. Front Endocrinol (Lausanne) 2019: 10858.

15. Bostrom P, Wu J, Jedrychowski MP, Korde A, Ye L, Lo JC, et al. A PGC1-alpha-dependent myokine that drives brown-fat-like development of white fat and thermogenesis. Nature 2012; 4817382: 463-8.

16. Roca-Rivada A, Castelao C, Senin LL, Landrove MO, Baltar J, Belen Crujeiras A, et al. FNDC5/irisin is not only a myokine but also an adipokine. PLoS One 2013; 84: e60563.

17. Anastasilakis AD, Polyzos SA, Saridakis ZG, Kynigopoulos G, Skouvaklidou EC, Molyvas D, et al. Circulating irisin in healthy, young individuals: day-night rhythm, effects of food intake and exercise, and associations with gender, physical activity, diet, and body composition. J Clin Endocrinol Metab 2014; 999: 3247-55.

18. Daskalopoulou SS, Cooke AB, Gomez YH, Mutter AF, Filippaios A, Mesfum ET, et al. Plasma irisin levels progressively increase in response to increasing exercise workloads in young, healthy, active subjects. Eur J Endocrinol 2014; 1713: 343-52.

19. Kraemer RR, Shockett P, Webb ND, Shah U, Castracane VD. A transient elevated irisin blood concentration in response to prolonged, moderate aerobic exercise in young men and women. Horm Metab Res 2014; 462: $150-4$.

20. Loffler D, Muller U, Scheuermann K, Friebe D, Gesing J, Bielitz J, et al. Serum irisin levels are regulated by acute strenuous exercise. J Clin Endocrinol Metab 2015: jc20142932.

21. Nygaard H, Slettalokken G, Vegge G, Hollan I, Whist JE, Strand T, et al. Irisin in blood increases transiently after single sessions of intense endurance exercise and heavy strength training. PLoS One 2015; 103: e0121367.

22. Hecksteden A, Wegmann M, Steffen A, Kraushaar J, Morsch A, Ruppenthal S, et al. Irisin and exercise training in humans - Results from a randomized controlled training trial. BMC Med 2013; 11235.

23. Scharhag-Rosenberger F, Meyer T, Wegmann M, Ruppenthal S, Kaestner L, Morsch A, et al. Irisin does not mediate resistance training-induced alterations in resting metabolic rate. Med Sci Sports Exerc 2014; 469: 1736-43.

24. Jedrychowski MP, Wrann CD, Paulo JA, Gerber KK, Szpyt J, Robinson MM, et al. Detection and quantitation of circulating human irisin by tandem mass spectrometry. Cell Metab 2015; 224: 734-40.

25. Fatouros IG. Is irisin the new player in exercise-induced adaptations or not? A 2017 update. Clin Chem Lab Med 2018; 564: 525-48.

26. Huh JY, Mougios V, Kabasakalis A, Fatouros I, Siopi A, Douroudos, II, et al. Exercise-induced irisin secretion is independent of age or fitness level and increased irisin may directly modulate muscle metabolism through AMPK activation. J Clin Endocrinol Metab 2014; jc20141437.

27. Qiu S, Cai X, Sun Z, Schumann U, Zugel M, Steinacker JM. Chronic exercise training and circulating irisin in adults: a meta-analysis. Sports Med 2015; 4511: 1577-88. 
28. Hew-Butler T, Landis-Piwowar K, Byrd G, Seimer M, Seigneurie N, Byrd B, et al. Plasma irisin in runners and nonrunners: no favorable metabolic associations in humans. Physiol Rep 2015; 31. https://doi.org/10. 14814/phy2.12262. Print 2015 Jan 1.

29. Singhal V, Lawson EA, Ackerman KE, Fazeli PK, Clarke H, Lee H, et al. Irisin levels are lower in young amenorrheic athletes compared with eumenorrheic athletes and non-athletes and are associated with bone density and strength estimates. PLoS One 2014; 96: e100218.

30. Bi J, Zhang J, Ren Y, Du Z, Li Q, Wang Y, et al. Irisin alleviates liver ischemia-reperfusion injury by inhibiting excessive mitochondrial fission, promoting mitochondrial biogenesis and decreasing oxidative stress. Redox Biol 2019: 20296-306.

31. Du J, Fan X, Yang B, Chen Y, Liu KX, Zhou J. Irisin pretreatment ameliorates intestinal ischemia/reperfusion injury in mice through activation of the Nrf2 pathway. Int Immunopharmacol 2019: 73225-35.

32. Zhang X, Hu C, Kong CY, Song P, Wu HM, Xu SC, et al. FNDC5 alleviates oxidative stress and cardiomyocyte apoptosis in doxorubicin-induced cardiotoxicity via activating AKT. Cell Death Differ 2019.

33. Hynynen E, Uusitalo A, Konttinen N, Rusko H. Heart rate variability during night sleep and after awakening in overtrained athletes. Med Sci Sports Exerc 2006; 382: 313-7.

34. Uusitalo AL, Vanninen E, Valkonen-Korhonen M, Kuikka JT. Brain serotonin reuptake did not change during one year in overtrained athletes. Int J Sports Med 2006; 279: 702-8.

35. Hynynen E, Uusitalo A, Konttinen N, Rusko H. Cardiac autonomic responses to standing up and cognitive task in overtrained athletes. Int J Sports Med 2008; 297: 552-8.

36. Kiviniemi AM, Tulppo MP, Hautala AJ, Vanninen E, Uusitalo AL. Altered relationship between R-R interval and R-R interval variability in endurance athletes with overtraining syndrome. Scand J Med Sci Sports 2014; 242: 77 .

37. Bluher S, Panagiotou G, Petroff D, Markert J, Wagner A, Klemm T, et al. Effects of a 1-year exercise and lifestyle intervention on irisin, adipokines, and inflammatory markers in obese children. Obesity (Silver Spring) 2014; 227: 1701-8.

38. Kreidler SM, Muller KE, Grunwald GK, Ringham BM, Coker-Dukowitz ZT, Sakhadeo UR, et al. GLIMMPSE: online power computation for linear models with and without a baseline covariate. J Stat Softw 2013; 5410.

39. Pekkala S, Wiklund PK, Hulmi JJ, Ahtiainen JP, Horttanainen M, Pollanen E, et al. Are skeletal muscle FNDC5 gene expression and irisin release regulated by exercise and related to health? J Physiol 2013; 591(Pt 21): 5393-400.

40. Huh JY, Panagiotou G, Mougios V, Brinkoetter M, Vamvini MT, Schneider BE, et al. FNDC5 and irisin in humans: I. Predictors of circulating concentrations in serum and plasma and II. mRNA expression and circulating concentrations in response to weight loss and exercise. Metabolism 2012; 6112: 1725-38.

41. Kurdiova T, Balaz M, Vician M, Maderova D, Vlcek M, Valkovic L, et al. Effects of obesity, diabetes and exercise on Fndc5 gene expression and irisin release in human skeletal muscle and adipose tissue: in vivo and in vitro studies. J Physiol 2014; 592(Pt 5): 1091-107.

42. Gmiat A, Mieszkowski J, Prusik K, Prusik K, Kortas J, Kochanowicz A, et al. Changes in pro-inflammatory markers and leucine concentrations in response to Nordic Walking training combined with vitamin D supplementation in elderly women. Biogerontology 2017; 184: 535-48.

43. Korkmaz A, Venojarvi M, Wasenius N, Manderoos S, Deruisseau KC, Gidlund EK, et al. Plasma irisin is increased following 12 weeks of Nordic walking and associates with glucose homoeostasis in overweight/obese men with impaired glucose regulation. Eur J Sport Sci 2019; 192: 258-66.

44. Carey AL, Steinberg GR, Macaulay SL, Thomas WG, Holmes AG, Ramm G, et al. Interleukin-6 increases insulin-stimulated glucose disposal in humans and glucose uptake and fatty acid oxidation in vitro via AMPactivated protein kinase. Diabetes 2006; 5510: 2688-97. 
45. Norheim F, Langleite TM, Hjorth M, Holen T, Kielland A, Stadheim HK, et al. The effects of acute and chronic exercise on PGC-1alpha, irisin and browning of subcutaneous adipose tissue in humans. FEBS J 2014; 2813: 739-49.

46. Askari H, Rajani SF, Poorebrahim M, Haghi-Aminjan H, Raeis-Abdollahi E, Abdollahi M. A glance at the therapeutic potential of irisin against diseases involving inflammation, oxidative stress, and apoptosis: an introductory review. Pharmacol Res 2018: 12944-55.

47. Kamenov Z, Assyov Y, Angelova P, Gateva A, Tsakova A. Irisin and testosterone in men with metabolic syndrome. Horm Metab Res 2017; 4910: 755-9.

48. Assyov Y, Gateva A, Karamfilova V, Gatev T, Nedeva I, Velikova T, et al. Impact of testosterone treatment on circulating irisin in men with late-onset hypogonadism and metabolic syndrome. Aging Male 2020: 1-7.

49. Ulker N, Yardimci A, Kaya Tektemur N, Bulmus O, Ozer Kaya S, Gulcu Bulmus F, et al. Irisin may have a role in pubertal development and regulation of reproductive function in rats. Reproduction 2020. 